\title{
An Evaluation of Childhood Tuberculosis Program in Chegutu District, Zimbabwe, 2020: A Descriptive Cross- Sectional Study
}

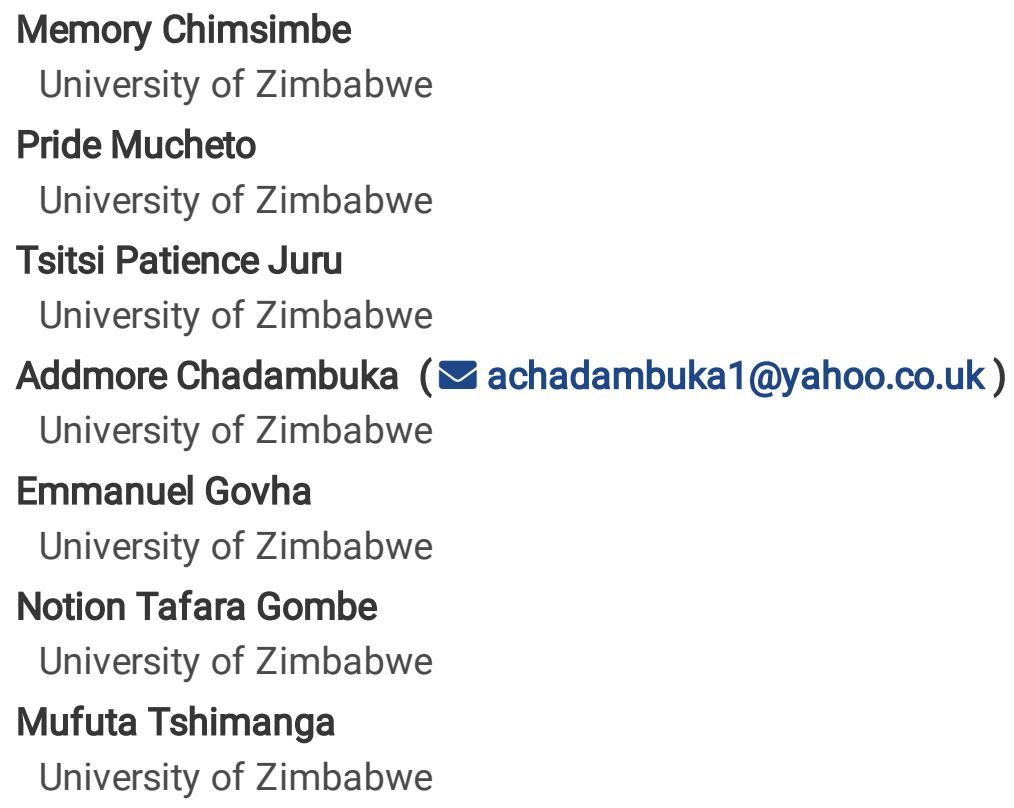

\section{Research Article}

Keywords: Childhood tuberculosis, program evaluation, Zimbabwe

Posted Date: December 1st, 2021

DOI: https://doi.org/10.21203/rs.3.rs-1067049/v1

License: (c) (1) This work is licensed under a Creative Commons Attribution 4.0 International License. Read Full License

Version of Record: A version of this preprint was published at BMC Health Services Research on April 14th, 2022. See the published version at https://doi.org/10.1186/s12913-022-07918-2. 


\section{Abstract \\ Background}

Childhood tuberculosis (TB) is a major global public health concern contributing to significant child morbidity and mortality. A records review of the TB notification for Chegutu District Health Information System 2 (DHIS2) showed a low childhood TB case detection rate. For 2018 and 2019, childhood TB notifications were 4\% and 7\% respectively against the national $12 \%$ case detection rate. We evaluated the performance of the childhood TB program in Chegutu.

\section{Methods}

We conducted a descriptive cross-sectional study. Sixty-six health workers (HW) participated in the study. Intervieweradministered questionnaires and checklists were used to collect data on reasons for low TB case detection, HW childhood TB knowledge, program inputs, processes and outputs. Strengths, Weaknesses, Opportunities and Threats analysis was used to assess the childhood TB processes. We analyzed the data using Epi Info $7^{\mathrm{TM}}$ to generate frequencies, proportions and means. A Likert scale was used to assess health worker knowledge.

\section{Results}

The majority $51 / 66(77 \%)$ of HW were nurses and $51 / 66(67 \%)$ of respondents were females. Reasons for the low childhood TB case detection were lack of HW confidence in collecting gastric aspirates 55/66(83\%) and HW's negative attitudes towards gastric aspirate collection 23/66(35\%). HW 24/66 (37\%) had a fair childhood TB notification knowledge. The district had only one functional X-ray machine for 34 health facilities. Only 6/18 motorcycles were functional with inadequate fuel supply. No desk guide for the management of TB in children for HW (2018) was available in 34 health facilities. Ethambutol 400mg was out of stock and adult $800 \mathrm{mg}$ tablets were used. Funds allocated for motor vehicle and motorcycles service (\$1612USD/year) were inadequate. The district failed to perform planned quarterly TB review meetings, contact tracing and childhood TB training due to funding and COVID-19 lockdown restrictions.

\section{Conclusion}

The childhood TB program failed to meet its targets due to inadequate inputs and suboptimal HW childhood TB knowledge. Case detection and notification can be improved through on-job training, mentorship, support and supervision and adequate resources.

\section{Background}

Childhood tuberculosis (TB) is a major public health problem that contributes to significant morbidity and mortality in children (1). Historically, tuberculosis has been neglected in children. The neglect can be explained by the difficulties in confirming a diagnosis of TB in children and the fact that children are not included in most surveys, poor recording and reporting practices, and misjudgement of tuberculosis in children as a low public health priority (2). 
Globally about half a million children were diagnosed and notified in both 2018 and 2019; the combined total of 1.04 million was $30 \%$ of the 5-year global target (for 2018-2022) of 3.5 million. Twenty-five per cent of the ten million people who were diagnosed with TB in 2019 were from the African region, with $10-20 \%$ of all TB cases expected to have occurred in children (3). In Zimbabwe, 25,775 people were diagnosed with TB in 2019, and about $10 \%$ of these were children below 15 years $(4,5)$. Despite TB being curable and preventable, nearly 650 children die from TB every day, with $80 \%$ of them dying before their fifth birthday (3).

Zimbabwe is a high TB burden country (3), and childhood TB notification is less than $10 \%$ annually with notification trends continuing on a downward spiral (5). The Government of Zimbabwe in its commitment to the control of TB adopted the Directly Observed Treatment, Short-course (DOTS) Strategy in 1994. Zimbabwe subscribes to the World Health Organisation (WHO) End TB strategy of reducing TB incidence by $90 \%$ by 2035 (5). A Desk Guide for Management of TB in children for health care workers was developed to improve TB management in children (6). Ministry of Health and Child Care (MOHCC) developed the Zimbabwe National Strategic TB Plan 2017-2020. One of the strategic objectives the nation is pursuing is; to increase the case detection and treatment of all forms of TB from $72 \%$ in 2015 to $85 \%$, with contribution from childhood TB increasing from $7-12 \%$ by 2020 (5).

A review of Chegutu District Health Information System (DHIS2) TB notification records showed that childhood tuberculosis case detection rate was low, in 2018 and 2019 tuberculosis notifications in children were 4\% and 7\% respectively against national childhood TB case detection of $12 \%$ by 2020 . Low case detection may imply that TB cases in children are missed, tuberculosis transmission and new infections will continue to rise unnoticed leading to childhood morbidity and mortality. The childhood TB notification in children of $12 \%$ by 2020 will not be met. The district was supported by Biomedical Research and Training Institute (BRTI) through the collection of sputum specimens from each facility twice weekly. Health care worker training on TB was done in 2018, 2019 and 2020. Ongoing TB mentorship, support and supervision were done in the district quarterly. Despite these efforts, childhood TB case detection remained low. There is no documented evidence to show that the childhood program in Chegutu was evaluated to assess its performance. We, therefore, evaluated the childhood tuberculosis program in the Chegutu District to make evidence-based recommendations to help improve the program.

\section{Methods}

\section{Study design}

We conducted a descriptive cross-sectional study based on the Centre for Disease Control and Prevention (CDC) logic model for program evaluation (7).

\section{Study Setting}

The study was conducted in Chegutu District, Mashonaland West Province situated in the central northern region of Zimbabwe. The district has 34 health facilities including a district hospital. It has a total population of 180741 people (8). Rural residents travel ten to twenty-five kilometres to access health care facilities. All government, rural and urban councils' health facilities were participating in the childhood TB program. The economic activities in the district consist of indigenous companies, mining, commercial farming and subsistence farming.

\section{Study population}

Our study population were health care workers from the outpatients' department, maternal child health department, pediatric wards, laboratory and pharmacy department. Caregivers of children being treated for TB in 2021 were 
interviewed. The District Medical Officer, the District Nursing Officer, Acting District TB Coordinator, District Environmental Health Officer, Senior Nursing Officer, District Pharmacist, Logistic Officer National Pharmaceutical Company and Laboratory Scientist were key informants in the study.

The TB notification forms, presumptive register and TB registers were reviewed for TB screening done, TB treatments done, contacts screened and TB notifications did. Laboratory stock cards were checked for TB consumables kits and Gene Xpert cartridges were ordered and received. Pharmacy ordering forms and stock cards were checked for childhood TB medicines ordered and received.

\section{Sample size for health care workers}

We calculated a sample size of 66 health care workers using the Dobson formula:

$\mathrm{n}=\mathrm{z}_{\mathrm{a}}{ }^{2} \times \mathrm{p}(1-\mathrm{p}) /$ delta ${ }^{2}$, assuming that $96 \%$ of the health workers knew that TB is curable from a study by Pantha et al. (2020) in Bangladesh (9) at 95\% confidence interval (Cl), $80 \%$ power, a margin of error of $5 \%$ and a non-response rate of $10 \%)$.

\section{Sample size for caregivers of children with TB}

Only five caregivers had children on TB treatment in the district during the time of data collection. We recruited the five caregivers into the study.

\section{Sampling of health care workers}

Health care workers from sixteen high volume sites and three hospitals in the district were recruited into the study. The 31 clinics in the Chegutu District have a staff complement of three nurses at each clinic. On the day of data collection, where three nurses reported for work, simple random sampling using a random number generated by the RANDBETWEEN function in Microsoft Excel was used to select two. If only one or two nurses reported for work, we recruited him or her into the study. At hospitals (Chegutu District, Mhondoro Rural and Norton) we randomly selected 12 health care workers from (out patient's department, opportunistic infection clinic, maternal child health department, paediatric ward, laboratory and pharmacy department) using random numbers generated by the RANDBETWEEN function in Microsoft Excel. Key informants were purposively recruited into the study.

\section{Sampling of caregivers}

Caregivers were conveniently recruited into the study.

\section{Data collection}

\section{Health care workers}

We collected data from health care workers using a pre-tested interviewer-administered questionnaire on demographic information, reasons for low childhood case detection, knowledge on childhood TB and processes involved in childhood TB. Presumptive registers, TB registers, notification forms, pharmacy ordering forms, laboratory ordering forms and stock cards and were reviewed to assess for childhood TB program outputs and outcome indicators. A checklist was used to assess childhood TB program resources availability.

\section{Key informants}


A key informant guide was used to collect data from the key informants on the childhood TB program budget, health care worker training that was conducted, availability of resources and performance of the program in 2020 . We used information collected from key informants to triangulate quantitative findings from health care workers, caregivers and childhood TB records.

\section{Caregivers of children with TB}

We used a pretested questionnaire to collect information from caregivers on their views regarding childhood TB.

\section{Data analysis}

We used Epi Info ${ }^{\mathrm{TM}} 7$ statistical software to capture and analyze data. Descriptive statistics were used to describe the study population and were presented as frequencies, proportions and median. Knowledge of health care workers was assessed using a 3-point Likert scale (good, fair, poor). Five questions were asked on childhood TB and scores were good for 4 and 5, fair knowledge for 3 and poor for 2 and below. An in-depth assessment of the TB program processes (childhood TB case finding, TB notification process TB contact tracing, the procurement and distribution process of childhood TB medicines and laboratory consumables) was done using the Strengths Weaknesses Opportunities and Threats (SWOT) analysis. Qualitative data from key informants were grouped manually into themes and was analysed for

\section{Results}

We interviewed sixty-six health care workers and five caregivers of children on TB treatment and the response rate was $100 \%$. The health care workers included nurses, doctors, environmental health technicians, pharmacy technicians, microscopists and laboratory technicians.

\section{Demographic characteristics of caregivers and health workers in childhood TB program in Chegutu District, 2020}

The majority $51 / 66(77 \%)$ of health care workers were nurses. Seventy-seven $(51 / 66)$ of the respondents were females. The median age of health care workers was 39 years $\left(Q_{1}=31 ; Q_{3}=57\right)$ and the median years in service of health care workers was 10 years $\left(Q_{1}=4 ; Q_{3}=14\right)$. The median age of caregivers was 44 years $\left(Q_{1}=40 ; Q_{3}=52\right)$ and the median age of children on TB treatment was 12 years $\left(Q_{1}=4 ; Q_{3}=14\right)$. Four out of the five children on TB treatment were males (Table 1).

\section{Reasons for low childhood TB case detection}

The reasons for the low childhood TB case detection reported by health care workers were lack of health care worker confidence in collecting gastric aspirates 55/66 (83\%), high workloads in health facilities $27 / 66$ (41\%), health care worker's negative attitudes towards gastric aspirate collection 23/66(35\%) and health care worker lack of knowledge on childhood TB 35/66 (35\%).

\section{Health care worker knowledge assessment on childhood TB program}

On knowledge assessment, the majority of the respondents 49/66 (74\%) knew that swollen lymph node was a common manifestation of extrapulmonary TB in children. Seventy per cent $46 / 66$ knew more than three key risk factors for TB in children. Sixty-seven per cent (44/66) of the respondents knew more than three investigations that are carried out for children who could not produce sputum. Fifty-five per cent $(36 / 66)$ of the respondents knew three 
or more symptoms of TB in children less than 10 years. Thirty per cent $(20 / 66)$ of the respondents knew that enlarged lymph nodes for suspected TB in children were two centimetres more in diameter. Using the 3-point Likert scale, healthcare workers had fair knowledge 24/66 (37\%) (Table 2).

\section{Caregivers' views of childhood TB program}

We interviewed five caregivers of children on TB treatment about their experiences in the process of the diagnosis and treatment of TB. One (1/5) caregiver highlighted that TB was diagnosed after two weeks of illness due to a lack of funds for a chest x-ray. Three (3/5) of the children had sputum collected for Gene Xpert within 24 hours of being a presumptive case. No medicine stock-outs were reported by caregivers, however, one (1/5) caregiver highlighted challenges with breaking Ethambutol tablets into two due to a shortage of pediatric TB formulations.

\section{Inputs injected into the childhood TB program in Chegutu District, 2020}

There were two functional Gene Xpert machines and one functional X-ray machine in the district for 34 health facilities. There was one non-functional motor vehicle for acquired immunodeficiency syndrome (AIDS) and TB program without fuel allocation to assist in the childhood TB program. The district had six out of 18 functional motorbikes that were responsible for the collection of specimens and distribution of results from and to the sites as well as contact tracing. There were three motorcycles from the Biomedical Research and Training Institute (BRTI) that supported the collection of sputum specimens from each facility twice weekly. All 34 health facilities did not have the Desk Guide for Management of TB in Children for Health Care Workers (2018). There were 10 ampoules of Tuberculin Skin Test (TST) which were in the pharmacy since March 2020 and had never been requested for use. Ethambutol $400 \mathrm{mg}$ was out of stock and adult tablets were being used. The funds allocated for servicing motor vehicle and motorcycles was inadequate (Table 3).

\section{SWOT analysis of the childhood TB processes}

\section{Case finding}

The district had two functional Gene Xpert machines, 50 Gene Xpert cartridges, 10 ampoules of Tuberculin skin tests and nine practising doctors. Twenty-two per cent (30/138) of the health care workers were trained in childhood TB which enabled childhood case detection. The turnaround time for sputum positive results was 24 hours. There was one functional X-ray machine for 34 health facilities in the district. There were no childhood TB management guidelines (2018) in the 17/17 health facilities visited.

\section{Notification}

All health facilities visited (17/17) had more than 20 TB notification forms which were adequate to notify the TB cases. Seventy-six per cent $(50 / 66)$ of the health workers were knowledgeable of the number of TB notification forms to complete during notification.

\section{Contact tracing}

There were more than 20 TB contact tracing forms in the 17 health facilities visited. There were 34 practising Environmental Health Technicians in the district. All 17/17 health facilities had cell phones loaded with airtime. There was inadequate fuel allocation (20litres/week) per motorcycle and a breakdown of 12 out of 18 motorcycles for contact tracing. 


\section{Ordering of childhood TB medicines}

Two health workers per facility were trained in Zimbabwe Assisted Pull System (ZAPS) and ordering based on consumption. The order fill rate of childhood TB medicines in 17/17 health facilities was below $100 \%$. There were stock-outs of childhood formulations especially Ethambutol in the 17/17 facilities.

\section{Ordering of laboratory consumables}

The order fill rate of TB consumables was $100 \%$. One health worker was hospital trained in the ordering of laboratory consumables. Ordering of laboratory consumables was based on consumption leading to no stocks out.

\section{Outputs and outcomes of the childhood TB program}

The proportion of children screened for TB in 2020 45/650(7\%) and the proportion of childhood TB cases detected was $21 / 539(4 \%)$ and the proportion of notified cases were $21 / 539(4 \%)$. All the children (21/21) had an HIV test done and the number of children who were HIV positive was 14/21. Only 9/14 HIV positive children were initiated on Antiretroviral Therapy (ART) (Table 4).

\section{Results from key informants}

\section{Resource availability}

We interviewed eight key informants involved in the childhood TB program in Chegutu District. The challenges that were highlighted by key informants included inadequate funds for motor vehicle and motorcycle servicing which led to repairs only. The district experienced fuel shortages for motorcycles, hindering contact tracing and specimen collection. Motorcycles were broken down which hindered transportation of sputum specimens and contact tracing. Staff attrition by experienced health workers was high. The district had no funds for conducting TB training and quarterly TB review meetings which are essential in identifying gaps and strengthening the TB program. One key informant highlighted stock-outs of childhood TB medicines leading to Ethambutol tablets being cut in two or four depending on the child's weight. The major issue from National Pharmaceuticals was the delay in the shipments of TB medicines resulting in stock-outs of some childhood TB medicines.

\section{Discussion}

We set out to evaluate the Childhood tuberculosis program in Chegutu District, and determine the reasons for the low detection of tuberculosis cases in children. The reasons for the low childhood TB case detection reported by the respondents were lack of health care worker confidence in the collection of gastric aspirates and lack of knowledge on childhood TB. Lack of confidence of health care workers could be attributed to lack of knowledge in conducting gastric aspirates. Hence, health workers need to be trained to boost their confidence. Similarly, a study by Oshi et al. (2016) in Nigeria revealed that health worker knowledge, and skills in TB detection in children was low. Nasogastric or nasopharyngeal intubation for collection of specimens was not done due to the negative attitude of the procedure by health workers leading to low case detection (10).

Health workers in Chegutu District had a fair knowledge of the childhood TB signs, symptoms, investigations and risk factors for childhood TB. Good knowledge plays a pivotal role in program implementation. Health care workers with good knowledge are confident in what they do. However, the health workers lacked knowledge on how to conduct gastric aspirates hence compromising their confidence to conduct the procedure. Similar findings were 
reported by Akma et al. (2020) in Malaysia where a knowledge gap on healthcare workers was noted on TB diagnosis resulting in low case detection (11).

The study revealed that there was inadequate funding for the childhood TB program. Limited funding resulted in poor maintenance of laboratory machinery such as the biological safety cabinet, microscopes and Gene Xpert machine, failure to repair X-ray machine, and failure to maintain a stable transportation system for sputum samples. Furthermore, the absence of vehicles for the TB control program hindered the district from conducting various activities such as delivering medicines in time or conducting supportive supervision of the health facilities involved in providing TB care. The whole district had one functional X-ray machine for 34 health facilities thereby reducing chances of clinical diagnosis of childhood TB. Resources play a key role in the success of a program. An interrupted supply of resources paralyses the program. It forms a barrier against achieving set targets. Similar findings were noted by Ereso et al. (2020) in Ethopia where inadequate resources led to low TB case finding and failure to carry out planned activities (12). This study showed that the childhood TB program had fuel shortages as it highly depended on donor funding. This affected transportation of specimens from sites and the conducting of contact tracing. Inadequate resources translate to strained processes, which in turn brings the low output of a program. TB and Leprosy Management Guidelines (2016) were available; however, the Desk-Guide for Management of TB in Children for Health Care Workers (2018) was not available in all visited health facilities. Guidelines are very important in programs as they provide step by step operating procedures for health workers. Similarly, Ereso et al. (2020) in Ethopia reported the absence of the national TB control program guideline in outpatient departments, laboratory rooms and TB rooms of the facilities leading to low case detection (12).

The processes in a program are interdependent with inputs that are injected. The process of childhood TB case detection, notification and contact tracing did not perform well in 2020. Quarterly TB review meetings did not take place due to lack of funding and COVID-19 pandemic restrictions. Reviewing a childhood TB program is crucial to assess how the program is performing to inform decision-making. Childhood TB cases detection performance remained below-set targets as inputs and processes that fed into the program were insufficient. In contrast to our findings, Malik et al. (2019) in Pakistan reported that adequate funding and resources played an important role in improving the performance of TB case detection (13). Our study revealed that ten tuberculin skin test ampoules expired in 2020 without being requested from the pharmacy. The ten doses that were ordered in March 2020 were not used till the end of 2020. Failure to conduct tuberculin skin tests by health workers could lead to low childhood TB case detection. Our findings are consistent with findings by Schwoebel et al. (2020) in a study in four African countries that reported that health workers had difficulties in administering and reading TST leading to non-use of TST for diagnosis of childhood TB (14).

In this study, the majority of caregivers reported that the children had symptoms of TB, such as cough, fever, weight loss and chest pain for one to two weeks before they were diagnosed as TB patients. The delay was mainly related to a referral from health centres to the district hospital for further investigation such as Chest X-ray which was paid for. Similar findings were reported by Pantha et al. (2020) in Bangladesh who noted that all four mothers reported that TB was suspected when other conditions were ruled out resulting in delays in TB treatment (9). Caregivers in our study reported the non-availability of a child-friendly regimen for the 4 Fixed-Dose Combination (FDC) combination (Isoniazid, Rifampicin, Ethambutol, Pyrazinamide). Similarly, Pantha et al. (2020) in Bangladesh noted that children who needed to take 4FDC had to take 3FDC and isoniazid which was not easy for children to take many tablets leading to poor compliance and poor treatment outcomes (9).

\section{Limitations}


Our study had some limitations. The beneficiaries of the childhood TB program who were interviewed in the study were caregivers of children diagnosed with TB; therefore, children's perspectives were not explored. We used convenience sampling for the caregivers hence the external validity of the findings presented is low. The study design was descriptive cross-sectional and the participants' answers were self-reported. Consequently, there may have been a possibility of interviewer bias and social desirability bias. To minimise social desirability bias, the respondents were only given a brief overview of the study from the start. This strategy helps to prevent the respondents from reacting in a particularly socially acceptable manner but creates space to fully explore their views and priorities before concentrating on the main topic of interest. To minimise interviewer bias, we used a wellstructured questionnaire for all participants in the comfort of their workplaces.

\section{Conclusions}

The childhood TB program failed to meet its targets due to inadequate inputs namely funds for vehicle and motorcycle servicing, fuel challenges and health care workers with fair knowledge of childhood TB. Apart from that, the district did not manage to perform the planned quarterly TB review meetings, contact tracing and childhood TB training due to lack of funding and COVID-19 lockdown restrictions. No processes, output and outcomes of the childhood TB program were met. Childhood TB case detection remained lower than the national target in 2020 (12\%) and needs improvement.

We recommended the integration of sputum specimen transportation with other programs such as the expanded program on immunisation. The district should conduct mentorship and on the job training on gastric aspirate collection, tuberculin skin test administration, sputum induction, stool collection for Gene Xpert and ongoing advocacy, communication and social mobilization activities to promote childhood TB. The District Medical Officer and the District Nursing Officer to address health workers negative attitudes towards gastric aspirates to improve sputum collection.

\section{Public health actions taken}

We discussed the importance of childhood TB with all interviewed health care workers and the results of the study were shared with Chegutu District Health Executive. They agreed to adopt the recommendations and enforce them in the district. We distributed 400 TB notification forms and distributed 400 contact tracing forms to the 34 health facilities

\section{List Of Abbreviations}

AIDS: Acquired Immunodeficiency Syndrome, BRTI: Biomedical Research and Training Institute, CDC: Centre for Disease Control and Prevention, CXR: Chest X-ray, DHIS: District Health Information System, DOTS: Directly Observed Treatment, Short-course, FDC: Fixed-Dose Combination, HCW: Health Care Worker, MOHCC: Ministry of Health and Child Care SWOT: Strengths, Weaknesses, Opportunities and Threats, TB: Tuberculosis, TST: Tuberculin Skin Test, WHO: World Health Organisation, ZAPS: Zimbabwe Assisted Pull System

\section{Declarations}

\section{Ethical approval and consent to participate}


The study was conducted following ethical principles. The Institutional Review Board (IRB) for Mashonaland West Health Directorate reviewed the study protocol and gave approval for the study. No participants' names or addresses were used during the study. Collected data was kept in privacy. Confidentiality was maintained throughout the study. Written informed consent was obtained from the study participants. Since data collection was conducted during the COVID-19 pandemic era, social distancing, hand hygiene and the wearing of a face mask covering the nose and mouth were maintained.

\section{Consent for publication}

Not applicable

\section{Availability of data and materials}

The data sets generated and analysed during the current study are available as supplementary files, Microsoft Access 2002-2003 (.mdb) [caregivers and programevaluation .mdb].

\section{Competing interests}

The authors declare that they have no competing interests.

\section{Funding}

No external funding was provided for the study. The authors funded the study using their savings.

\section{Author's contributions}

MC, PM, TPJ, AC, EG, NTG, and MT: conception, design, and acquisition of data. MC, PM, TPJ, conducted data analysis and interpretation of data. MC, PM, TPJ, wrote the first draft of the manuscript. TPJ, AC, EG, NTG, and MT: reviewed several drafts of the manuscript for intellectual content. All authors approved the final version of the manuscript

\section{Acknowledgements}

We would like to acknowledge the following for making our study a success: Provincial Directorate Mashonaland West, District Medical Offices Chegutu District and Health Studies Office: University of Zimbabwe and the study participants.

\section{References}

1. World Health Organization, International Union against Tuberculosis and Lung Disease. Childhood TB training toolkit [Internet]. Geneva: World Health Organization; 2014 [cited 2021 Nov 8]. 39 p. Available from: https://apps.who.int/iris/handle/10665/134387

2. Seddon JA, Jenkins HE, Liu L, Cohen T, Black RE, Becerra MC, et al. Counting Children with Tuberculosis: Why Numbers Matter. Int J Tuberc Lung Dis Off J Int Union Tuberc Lung Dis [Internet]. 2015 Dec [cited 2021 Jun 29];19(0 1):9-16. Available from: https://www.ncbi.nlm.nih.gov/pmc/articles/PMC4708268/

3. World Health Organization. GLOBAL TUBERCULOSIS REPORT 2020 [Internet]. World Health Organization; 2020 [cited 2021 Nov 8]. Available from: https://apps.who.int/iris/bitstream/handle/10665/336069/9789240013131eng.pdf 
4. Zimbabwe is on track to achieve the 2020 Global End TB Strategy milestones [Internet]. WHO | Regional Office for Africa. [cited 2021 Nov 8]. Available from: https://www.afro.who.int/news/zimbabwe-track-achieve-2020global-end-tb-strategy-milestones-0

5. National Tuberculosis Program -Strategic Plan (2017-2020). [cited 2021 Nov 8];97. Available from: https://depts.washington.edu/edgh/zw/hit/web/project-resources/TB-NSP.pdf

6. Desk_Guide_Management_TB_Children_Zimbabwe.pdf [Internet]. [cited 2021 Nov 8]. Available from: https://www.challengetb.org/publications/tools/country/Desk_Guide_Management_TB_Children_Zimbabwe.pdf

7. cdc-logic-model-development.pdf [Internet]. [cited 2021 Nov 8]. Available from: https://www.utep.edu/orsp/reas/_Files/docs/cdc-logic-model-development.pdf

8. Chikeya L. DISTRICT POPULATION PROJECTIONS. [cited 2021 Nov 8];75. Available from: https://www.zimstat.co.zw/wp-content/uploads/publications/Population/population/DistrictProjections/District-Population-Projection-Report-Mashonaland-west.pdf

9. Pantha S, Aguinaldo MJ, Bari SMH, Chowdhury S, Dendup U, Gupta RD, et al. Facilitators and Barriers to Implementation of Childhood Tuberculosis Control Program in Bangladesh: A Mixed-Methods Study from BRAC Urban DOTS Centres in Dhaka [Internet]. 2021 [cited 2021 Nov 8]. Available from:

https://www.researchsquare.com/article/rs-39360/v1

10. Oshi DC, Chukwu JN, Nwafor CC, Meka AO, Madichie NO, Ogbudebe CL, et al. Does intensified case finding increase tuberculosis case notification among children in resource-poor settings? A report from Nigeria. Int J Mycobacteriology [Internet]. 2016 Mar 1 [cited 2021 Nov 8];5(1):44-50. Available from:

https://www.sciencedirect.com/science/article/pii/S2212553115001612

11. Akma N, Mustafa M, Suran SA, Marwan AA, Omar MR. Knowledge, attitude, practice and stigma of tuberculosis among healthcare providers in hospital Sultan Haji Ahmad Shah, Temerloh, Pahang. Int J Res Pharm Sci [Internet]. 2019 Nov 7 [cited 2021 Nov 8];10(SPL1). Available from: https://www.pharmascope.org/index.php/ijrps/article/view/1679

12. Ereso BM, Yimer SA, Gradmann C, Sagbakken M. Barriers for tuberculosis case finding in Southwest Ethiopia: $A$ qualitative study. PLOS ONE [Internet]. 2020 Jan 2 [cited 2021 Nov 8];15(1):e0226307. Available from: https://journals.plos.org/plosone/article?id=10.1371/journal.pone.0226307

13. Malik AA, Hussain H, Creswell J, Siddiqui S, F. Ahmed J, Madhani F, et al. The Impact of Funding on Childhood TB Case Detection in Pakistan. Trop Med Infect Dis [Internet]. 2019 Dec 15 [cited 2021 Nov 8];4(4):146. Available from: https://www.ncbi.nlm.nih.gov/pmc/articles/PMC6958435/

14. Schwoebel V, Koura KG, Adjobimey M, Gnanou S, Wandji AG, Gody J-C, et al. Tuberculosis contact investigation and short-course preventive therapy among young children in Africa. Int J Tuberc Lung Dis [Internet]. 2020 Apr 1 [cited 2021 Nov 7];24(4):452-60. Available from:

https://www.ingentaconnect.com/content/iuatld/ijtld/2020/00000024/00000004/art00017

\section{Tables}

Table 1: Demographic characteristics of health workers and caregivers in childhood TB program, Chegutu District, 2020 


\begin{tabular}{|c|c|c|c|}
\hline Variable & Category & Frequency $(n=66)$ & Percentage \\
\hline \multirow[t]{2}{*}{ Sex } & Female & 51 & 77 \\
\hline & Male & 15 & 23 \\
\hline \multirow[t]{2}{*}{ Age } & & Median age $=39$ years & \\
\hline & & $Q_{1}=31 ; Q_{3}=57$ & \\
\hline \multirow[t]{6}{*}{ Designation } & Nurses & 51 & 77 \\
\hline & Environmental Health Technician & 5 & 8 \\
\hline & Doctors & 4 & 6 \\
\hline & Laboratory Technician & 3 & 5 \\
\hline & Pharmacy Technicians & 2 & 3 \\
\hline & Microscopist & 1 & 1 \\
\hline \multirow[t]{2}{*}{ Years in service } & & Median: 10 years & \\
\hline & & $Q_{1}=4 ; Q_{3}=14$ & \\
\hline Caregivers & & 5 & \\
\hline \multirow[t]{2}{*}{ Sex of child } & Male & 4 & \\
\hline & Female & 1 & \\
\hline
\end{tabular}

Age of child in years

Median: 12 years

$\mathrm{Q}_{1}=4 ; \mathrm{Q}_{3}=14$

Table 2: Health worker knowledge assessment of the childhood TB program, Chegutu District, 2020

\begin{tabular}{lll} 
Variable & $\begin{array}{l}\text { Frequency } \\
\text { n=66 }\end{array}$ & Perce \\
$\begin{array}{l}\text { Know that lymph node was a common manifestation of Extrapulmonary TB in } \\
\text { children }\end{array}$ & 49 & 74 \\
\hline Know the key risk factors for TB in children & 46 & 70 \\
\hline Know the investigations done for children who cannot produce sputum & 44 & 67 \\
\hline $\begin{array}{l}\text { Know the main symptoms of pulmonary childhood TB in children less than } 10 \\
\text { years }\end{array}$ & 36 & 55 \\
\hline
\end{tabular}


3- point Likert scale (Good, fair, poor)

\begin{tabular}{lll} 
Fair & 24 & 37 \\
Good & 22 & 33 \\
\hline Poor & 20 & 30
\end{tabular}

Table 3: Inputs injected into the Childhood TB program, Chegutu District, 2020 
Item

Funds for TB vehicle servicing

Funds for motorcycle servicing

\$806USD / year

\$4000USD/year

\section{Comment}

$\$ 4000 U S D / y e a r$

Funds were not adequate for servicing; money was only used for repairs

\begin{tabular}{|c|c|c|c|}
\hline Fuel & $\begin{array}{l}20 \text { litres/week for a } \\
\text { bike }\end{array}$ & $\begin{array}{l}\text { 40litres a week } \\
\text { for a bike }\end{array}$ & $\begin{array}{l}\text { Inadequate fuel for specimen } \\
\text { collection and contact } \\
\text { tracing. }\end{array}$ \\
\hline Vehicle & 1 & 2 & $\begin{array}{l}\text { The vehicle was non- } \\
\text { functional in } 2020\end{array}$ \\
\hline Motorcycles & 18 & $\begin{array}{l}1 / \text { health } \\
\text { facility }=34\end{array}$ & $\begin{array}{l}\text { Only } 6 \text { motor cycles were } \\
\text { functional in } 2020\end{array}$ \\
\hline TB consumable kit & 12kits & 12kits & Adequate \\
\hline Gene Xpert cartridges & $15 \times 50$ cartridges & $\begin{array}{l}15 \times 50 \\
\text { cartridges }\end{array}$ & Adequate \\
\hline $\begin{array}{l}\text { Rifampicin/Isoniazid/ } \\
\text { Pyrazinamide }\end{array}$ & $\begin{array}{l}10 \text { tins } \times 84 \text { tablets } \\
\text { (above minimum } \\
\text { stock) }\end{array}$ & $\begin{array}{l}\text { 12tins } \times 84 \\
\text { tablets }\end{array}$ & Adequate \\
\hline Rifampicin/Isoniazid & $\begin{array}{l}10 \text { tins } \times 84 \text { tablets } \\
\text { (above minimum } \\
\text { stock) }\end{array}$ & $\begin{array}{l}12 \text { tins } \times 84 \\
\text { tablets }\end{array}$ & Adequate \\
\hline
\end{tabular}

Isoniazid 100mg

20 tins $\times 100$ tablets

12 tins $\times 100$

Above maximum stock tablets

Funds were not adequate for servicing; money was only used for repairs collection and contact

The vehicle was non-

nly 6 motor cycles were $1 /$ health functional in 2020

Adequate stock)

12 tins $\times 84 \quad$ Adequate (ablets

Ethambutol 400mg

0

2 tins $x 1000$

Using adult's tablets tablets

Desk Guide for the management of 0 TB in Children for Health Workers (2018)

Tuberculin skin test

National TB/HIV management
$1 /$ health facility

10 ampoules

10 ampoules

2/health facility

Not available in all health facilities.

Were adequate. Not requested from the pharmacy since March 2020.

1/health facility Were adequate in the district 
guidelines

TB screening tools

4/ health facility 4/ health facility

Only adult TB screening tools

Gene Xpert machines

1 machine (4 specimens/2hours)

TB notification forms were available

One machine had only 3 modules functional instead of 4

1 machine (3 specimens/ 2 hours)

X-ray machine 1 2 Functional

Table 4: Outputs and Outcomes in the Childhood TB program in Chegutu District,2020

\begin{tabular}{llll}
\hline Outputs & Achieved & Target & Comments \\
\hline $\begin{array}{l}\text { Number of childhood TB cases } \\
\text { detected }\end{array}$ & $\begin{array}{l}21 / 539 \\
(4 \%)\end{array}$ & $12 \%$ & The district did not reach its target \\
\hline $\begin{array}{l}\text { Number of childhood TB cases } \\
\text { notified }\end{array}$ & $\begin{array}{l}21 \\
/ 539(4 \%)\end{array}$ & $12 \%$ & The district did not reach its target \\
$\begin{array}{l}\text { Number of children initiated on } \\
\begin{array}{l}\text { TB treatment } \\
\text { Number of children with HIV test }\end{array}\end{array}$ & $21 / 539(4 \%)$ & $12 \%$ & The district did not reach its target \\
\hline $\begin{array}{l}\text { Nesults } \\
\text { resuld }\end{array}$ & $100 \%$ & The district did not reach its target \\
\hline
\end{tabular}

Number of children HIV positive $\quad$ 14/21

Thirty-two per cent of the children were HIV negative

Number of children on ART

9/14 $100 \% \quad$ Five of the children were not put on ART as they were still within two weeks of commencement of TB treatment

Number of contacts initiated on $\quad 28 / 28$ $100 \%$ Under-fives who were commenced on IPT were all Isoniazid Preventive Therapy from adults as index TB cases 